\title{
Aortic Valve Stenosis with Black Pigmentation Due to Alkaptonuria: A Case Report
}

\author{
Takahiro Shojima ${ }^{1}$, Yasuyuki Zaima ${ }^{2}$, Kazuyoshi Takagi ${ }^{3}$, Tohru Takaseya ${ }^{4}$, Hiroyuki \\ Otsuka $^{5}$, Yusuke Shintani ${ }^{5}$, Takanori Kono ${ }^{4}$, Satoshi Kikusaki ${ }^{1}$, Kosuke Saku ${ }^{2}$, and Eiki \\ Tayama ${ }^{4}$ \\ ${ }^{1}$ Kurume University School of Medicine \\ ${ }^{2}$ Kurume University Hospital \\ ${ }^{3}$ Kurume university, school of medicine \\ ${ }^{4}$ Affiliation not available \\ ${ }^{5}$ Munakata Suikokai General Hospital
}

April 22, 2021

\begin{abstract}
We report about a 78-year-old woman with severe aortic valve stenosis (AS). She had heavily calcified and thickened aortic valve leaflets with black pigmentation. After excision of the aortic valve leaflets, biological aortic valve replacement (AVR) was performed. Alkaptonuria was diagnosed and confirmed based on increased urinary excretion of homogentisic acid. She has had no cardiovascular system-related symptoms for 3 years and no evidence of structural valve deterioration. Data on the long-term outcomes of AVR associated with structural valve deterioration that could regulate prosthetic valve selection in alkaptonuria are limited. Therefore, further research on the natural evolution of AS and the rate of structural valve deterioration after AVR is needed to provide an optimal prosthesis for these patients.
\end{abstract}

\section{INTRODUCTION}

Alkaptonuria is an autosomal recessive disease caused by a deficiency of homogentisic acid 1,2-dioxygenase that leads to homogentisic acid (HGA) accumulation. Its clinical signs include dark urine, melanin-like pigmentation of tissues (ochronosis), and ochronotic arthropathies. HGA accumulation also affects the cardiovascular system, especially the heart valves. Alkaptonuria cannot be diagnosed preoperatively since most of the clinical signs are confirmed intraoperatively.

We present a rare case of aortic valve stenosis (AS) with black pigmentation caused by alkaptonuria that was diagnosed postoperatively. Cardiac surgeons should recognize this condition when they observe typical signs during surgery.

\section{CASE PRESENTATION}

A 78-year-old woman was referred to our institute for an elective AVR for AS. At 55 years of age, she had undergone a left hip replacement for degenerative arthropathy. She had consanguineous parents. Transthoracic echocardiography showed a thickened and calcified aortic valve with a mean transvalvular gradient and calculated aortic valve area of $57.4 \mathrm{mmHg}$ and $0.63 \mathrm{~cm}^{2}$, respectively. Cardiac catheterization revealed severe AS with a transvalvular pressure gradient of $99 \mathrm{mmHg}$ and no calcification or stenosis of coronary vessels. The patient underwent AVR for severe asymptomatic AS.

A cardiopulmonary bypass was performed intraoperatively using aortic and caval cannulation, and moderate 
hypothermia was induced. Cold antegrade crystalloid cardioplegia was performed. After an oblique aortotomy, black pigmentation of the aortic intima extending to the aortic valve leaflet was observed (Figure 1). The leaflets were heavily calcified, thickened, and covered with black patches. The black pigmentation extended to the left ventricular outflow tract and anterior leaflet of the mitral valve. AVR was performed using a 19-mm bioprosthesis (Crown PRT, LivaNova, London, UK).

Postoperatively, the patient disclosed a history of normal-colored urine on voiding, which darkened in diapers during her childhood. Her sclerae and cartilage of the pinnae were bluish black. Discoloration of collagenous tissues and a history of degenerative arthropathy and dark urine prompted the diagnosis of alkaptonuria. Increased urinary excretion of HGA confirmed this diagnosis. A histopathological examination of the excised aortic valve leaflets revealed dark-brown pigmentation in the body of the valve, which was associated with calcium nodules (Figure 2). The postoperative period was uneventful. She was discharged from the hospital without complications. She has been free from any cardiovascular system-related symptoms for 3 years. Follow-up echocardiography revealed a mean prosthetic valve gradient, calculated aortic valve area, and left ventricular ejection fraction of $9.7 \mathrm{mmHg}, 1.53 \mathrm{~cm}^{2}$, and $72 \%$, respectively. The difference in prosthetic valve functionality between discharge and follow-up was not significant.

\section{DISCUSSION}

Alkaptonuria occurs in 1/250,000-1/1,000,000 people. ${ }^{1}$ In consanguineous groups, the incidence may be as high as $1 / 25,000{ }^{2}$ The enzyme deficiency prevents the breakdown of HGA to fumaric and acetoacetic acids, resulting in an increased concentration of HGA in the urine and many tissues. This causes the classic clinical triad of dark urine at birth, ochronosis, and degenerative ochronotic arthropathy. The effect of HGA accumulation on the cardiovascular system is usually discovered at 60-70 years of age. Alkaptonuria often remains undiagnosed in childhood because urine discoloration can only be detected in urine that has been left standing. Furthermore, because of the lack of specific symptoms, it is diagnosed postoperatively due to arthropathy or valvular disease.

No guidelines exist for optimal prosthetic valve selection for AVR. Because the bioprostheses may be exposed to ochronotic pigment-related valvular calcification like the native valve tissue, several case reports have recommended the use of mechanical prostheses. ${ }^{3,4,5}$ However, structural valve deterioration due to ochronosis in bioprostheses has not been described, and only one study reported on the functionality of the bioprosthetic valve after the patient was discharged. ${ }^{6}$ For this patient, we chose a bioprosthesis preoperatively considering her age and surgical risks and benefits. Follow-up echocardiography performed 3 years postoperatively revealed no evidence of structural valve deterioration. It may take time for the prosthetic valve to deteriorate because the ochronotic pigment must accumulate. Further studies involving more cases are needed to determine the optimal choice of prostheses in patients with alkaptonuria.

Although there is no standard treatment for alkaptonuria, the prognosis is relatively good. Currently, the treatment is only palliative. ${ }^{7}$ Nitisinone inhibits 4-hydroxyphenylpyruvate dioxygenase, the enzyme that causes HGA formation, dramatically reducing the production and urinary excretion of HGA. ${ }^{8}$ Taking 2 $\mathrm{mg}$ /day of nitisinone slows the clinical progression of alkaptonuria, decreases HGA levels, and arrests ochronosis, the cause of tissue damage in alkaptonuria. ${ }^{9}$ Therefore, nitisinone may be one of the safest treatments to prevent the progression of calcification in the native aortic or bioprosthetic valve. However, due to limited data on aortic valve disease, we did not use this drug in our patient. Further studies are needed to determine the safety and long-term efficacy of long-term nitisinone use when treating alkaptonuria.

In conclusion, alkaptonuria should be considered when tissue pigmentation and arthritis are present, along with valvular disease and coronary artery calcification. Routine echocardiography is recommended in patients older than 50 years diagnosed with alkaptonuria. The short-term outcomes were good, but data on the longterm outcomes that could help regulate the selection of prosthetic valves to treat AS are limited. Thus, further research is needed on the selection of a prosthesis in patients with alkaptonuria and to understand the natural history of AS and the rate of structural valve deterioration after bioprosthesis AVR.

\section{AUTHOR CONTRIBUTIONS}


Drafting article: T.S.

Critical revision of article: E.T.

Approval or article: Y.Z., K.T., T.T., H.O., Y.S., T.K., S.K., K.S.

REFERENCES

1. Phornphutkul C, Introne WJ, Perry MB, et al. Natural history of alkaptonuria. N Engl J Med 2002;347:2111-2121.

2. Albers SE, Brozena SJ, Frank GL, Fenske NA. Alkaptonuria and ochronosis: Case report and review. J Am Acad Dermatol 1992;27:609-614.

3. Thakur S, Markman P, Cullen H. Choice of valve prosthesis in a rare clinical condition: Aortic stenosis due to alkaptonuria. Hear Lung Circ 2013;22:870-872.

4. Atalay A, Gocen U, Basturk Y, Kozanoglu E, Yaliniz H. Ochronotic involvement of the aortic and mitral valves in a 72-year-old man. Texas Heart Inst J 2015;42:84-86.

5. Atalay A, Polat V, Turhan N Tasoglu I. A rare cause of aortic valve stenosis: Ochronosis. Turkish J Thorac Cardiovasc Surg 2018;261:467.

6. Capuano F, Emiliano A, Antonino R, et al. Blackish pigmentation of the aorta in patient with alkaptonuria and Heyde's syndrome. Aorta (Stamford) 2014;2:74.

7. Ranganath LR, Jarvis JC, Gallagher JA. Recent advances in management of alkaptonuria (invited review; Best practice article). J Clin Pathol 2013;66:367-373.

8. Introne WJ, Monique BP, James T, et al. A 3-year randomized therapeutic trial of nitisinone in alkaptonuria. Mol Genet Metab 2011;103:307-314.

9. Ranganath LR, Khedr M, Milan AM, et al. Nitisinone arrests ochronosis and decreases rate of progression of Alkaptonuria: Evaluation of the effect of nitisinone in the United Kingdom National Alkaptonuria Centre. Mol Genet Metab 2018;125:127-134.

\section{FIGURE LEGENDS}

\section{FIGURE 1}

Macroscopic findings. A. Intraoperative findings showing black pigmentation in the aortic lumen and aortic valve leaflet. B. Black pigmentation extending to the left ventricular outflow tract and anterior leaflet of the mitral valve (arrow) after aortic valve leaflet excision. C. Resected aortic valve leaflets. The leaflets have black patches with calcification, but the edges of the cusps are relatively free from degenerative changes.

FIGURE 2

Histopathological examination of the excised aortic valve leaflets showing dark-brown pigment deposition in the valve cusps, associated with calcium nodules. 
(A)

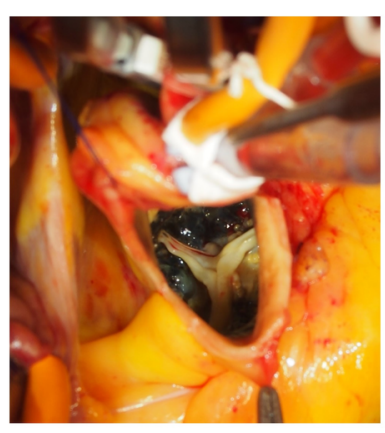

(B)

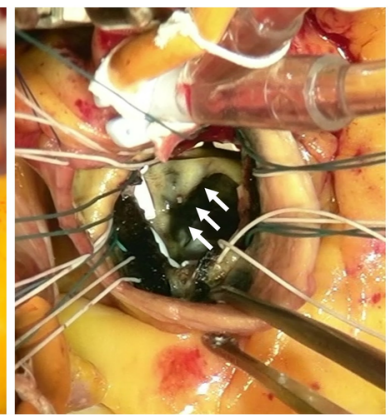

(C)

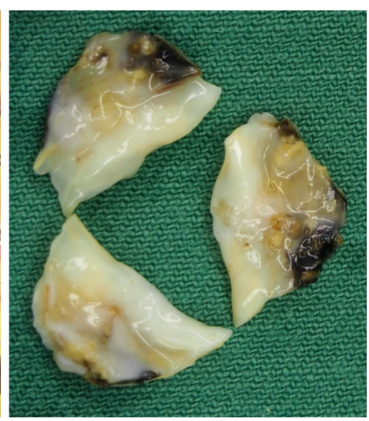

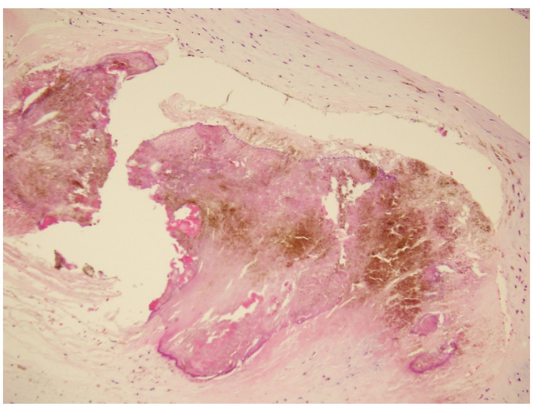

